MITSUBISHI ELECTRIC RESEARCH LABORATORIES

http://www.merl.com

\title{
Super-Resolution Blind Channel Modeling
}

\author{
Pun, M-O.; Molisch, A.F.; Orlik, P.; Okazaki, A.
}

TR2011-028 June 2011

\begin{abstract}
We consider the problem of extracting a wide-band channel model when only measurements in parts of this band are available, specifically in disjoint frequency sub-bands. Conventional channel modeling techniques cannot model at all those parts of the band where no sounding signals are available; or, if they use conventional interpolation, suffer from poor performance. To circumvent this obstacle, we develop in this paper a three-step super-resolution blind algorithm. First, the path delays are estimated by exploiting super-resolution algorithms such as MUSIC or ESPRIT based on the transfer function of each sub-band, separately. Exploiting such a set of delay estimates, the proposed algorithm performs blind (i.e., without training signal) channel estimation over the unmeasured sub-bands, and subsequently derives the frequency response over the whole wide-band channel. Finally, estimates derived from different sub-bands are combined via a soft combining technique. Computer simulations show that the proposed super-resolution blind algorithm can achieve a significant performance gain over conventional methods.
\end{abstract}

IEEE International Conference on Communications (ICC)

This work may not be copied or reproduced in whole or in part for any commercial purpose. Permission to copy in whole or in part without payment of fee is granted for nonprofit educational and research purposes provided that all such whole or partial copies include the following: a notice that such copying is by permission of Mitsubishi Electric Research Laboratories, Inc.; an acknowledgment of the authors and individual contributions to the work; and all applicable portions of the copyright notice. Copying, reproduction, or republishing for any other purpose shall require a license with payment of fee to Mitsubishi Electric Research Laboratories, Inc. All rights reserved.

Copyright (C) Mitsubishi Electric Research Laboratories, Inc., 2011

201 Broadway, Cambridge, Massachusetts 02139 



\title{
Super-Resolution Blind Channel Modeling
}

\author{
Man-On Pun ${ }^{\dagger}$, Andreas F. Molisch ${ }^{\ddagger}$, Philip Orlik ${ }^{\dagger}$ and Akihiro Okazaki ${ }^{\sharp}$ \\ $\dagger$ Mitsubishi Electric Research Laboratories (MERL), Cambridge, MA 02139, USA \\ ${ }^{\ddagger}$ Ming Hsieh Depart. of Electrical Engineering, University of Southern California, Los Angeles, CA 80089, USA \\ ${ }^{\sharp}$ Mitsubishi Electric Corporation, 5-1-1 Ofuna Kamakura, Kanagawa 2478501, Japan.
}

\begin{abstract}
We consider the problem of extracting a wideband channel model when only measurements in parts of this band are available, specifically in disjoint frequency subbands. Conventional channel modeling techniques cannot model at all those parts of the band where no sounding signals are available; or, if they use conventional interpolation, suffer from poor performance. To circumvent this obstacle, we develop in this paper a three-step super-resolution blind algorithm. First, the path delays are estimated by exploiting super-resolution algorithms such as MUSIC or ESPRIT based on the transfer function of each subband, separately. Exploiting such a set of delay estimates, the proposed algorithm performs blind (i.e., without training signal) channel estimation over the unmeasured subbands, and subsequently derives the frequency response over the whole wideband channel. Finally, estimates derived from different subbands are combined via a soft combining technique. Computer simulations show that the proposed super-resolution blind algorithm can achieve a significant performance gain over conventional methods.
\end{abstract}

Index Terms-Super-resolution channel modeling, blind channel estimation, soft combining.

\section{INTRODUCTION}

Accurate characterization of wireless propagation channels plays a critical role in designing high-performance wireless systems. As demonstrated in Shannon's seminal work, the fundamental performance limits of wireless transmission are dictated by the wireless channel characteristics. Hence, an indepth understanding of the underlying channel can facilitate system architects to design, optimize and subsequently analyze practical wireless systems [1], [2].

For the purpose of system development, channel models based on measurements are essential. Conventionally, channel measurements are conducted by sending and measuring sounding signals over the whole frequency band of interest. However, there are often challenging situations in which sounding signals can be transmitted only over some parts of the frequency band of interest, rather than the whole band. Such challenges arise in a number of practical situations including regulatory restrictions, measurements with interference and reuse of narrowband measurements.

First of all, as existing (legacy) wireless services such as analog TV broadcasting are eliminated or relocated from particular frequency bands, the freed-up bands may be re-grouped to provide various broadband services. Thus, channel models for these wideband channels are required to develop future applications even before the legacy services are terminated. However, measurements of the channel characteristics can only be performed in the "whitespace" between the existing channels while the legacy services are still operating. Secondly, for many measurements, it is impossible to guarantee absence of interference over the whole desired bandwidth, which is particularly true for ISM (Industrial, Scientific, and Medical) bands due to their license-free operation. Traditionally, all measurements contaminated by interference have to be discarded, despite the fact that the bandwidth of the interference is often smaller than the measurement bandwidth. Given the high cost incurred during channel measurements, it is thus highly desirable if channel models can be directly derived from the interference-free measurements over some parts of the desired frequency band. Thirdly, each generation of wireless data system occupies more bandwidth than the previous one, and needs therefore more broadband channel models. While such broadband channel models can be derived through new measurement campaigns, the enormous efforts incurred make it worthwhile to investigate whether or not existing narrowband measurements in adjacent frequency bands can be reused.

Thus motivated, a natural question to ask is whether it is feasible to measure a wideband wireless channel by sending only narrowband sounding signals. In this work, we demonstrate that this task can be successfully achieved. Taking advantage of the proposed super-resolution blind algorithm, we show that a wideband wireless channel can be accurately measured and modeled using narrowband sounding signals transmitted in disjoint subbands. More specifically, by exploiting a parametric channel model to extract channel information such as multipath component (MPC) delays and amplitudes, we propose to estimate the channel transfer function that is valid over a larger bandwidth than the original measurements. Furthermore, since this estimation can be performed in each separate subband, estimations derived from different subbands are then weighted and combined for channel model extraction.

The main contributions of this paper are as follows: we present the (to the best of our knowledge) first algorithm that obtains consistent channel measurements from measurements in disjoint subbands (separated by more than a coherence bandwidth of the channel). Furthermore, this work explicitly incorporates the effects of practical pulse shaping filters (raised-cosine filters) into the high-resolution algorithms such as multiple signal classification (MUSIC) algorithm or frequency-domain Estimation of Signal Parameters via Rotational Invariance Technique (ESPRIT), which distinguishes this work from other applications of these high-resolution algorithms for channel estimation such as [3], [4]. Finally, 


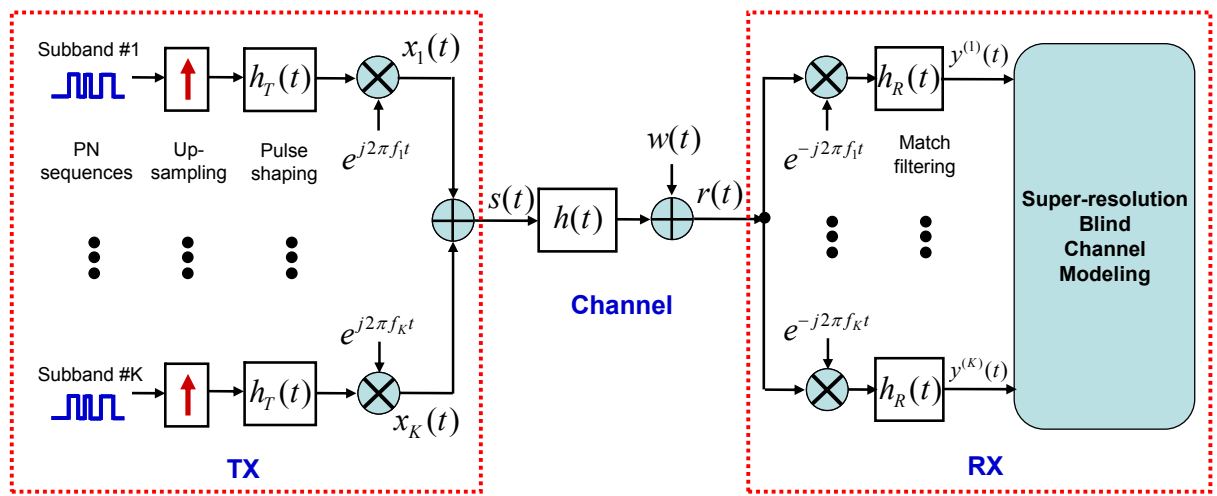

Fig. 1. Block diagram of the measurement system under consideration.

unlike [3], [4] that solely concentrate on path delay extraction, our work further characterizes the MPC amplitudes and subsequently the channel transfer function over a larger bandwidth.

Notation: Vectors and matrices are denoted by boldface letters. $(\cdot)^{\dagger},(\cdot)^{T}$ and $(\cdot)^{H}$ stand for the Moore-Penrose pseudoinverse, transpose operation and Hermitian transposition, respectively. $|\cdot|$ denotes the amplitude of the enclosed complexvalued quantity while $\lfloor x\rfloor$ is the maximum integer less than $x$. Furthermore, $[\boldsymbol{A}]_{i, j}$ denotes the $i$-th row and $j$-th column entry of matrix $\boldsymbol{A}$ whereas $\boldsymbol{A}(q,:)$ the $q$-th column of matrix $\boldsymbol{A}$. Finally, $\boldsymbol{I}_{N}$ is the $N \times N$ identity matrix while $\boldsymbol{F}_{N}$ is the $N$-point discrete Fourier transform (DFT) matrix with entries $[\boldsymbol{F}]_{n, k}=\frac{1}{\sqrt{N}} \exp \left(\frac{-j 2 \pi n k}{N}\right)$ for $0 \leq n, k \leq N-1$.

\section{Signal Model}

We consider a channel measurement system shown in Fig. 1. The system consists of $K$ disjoint narrowband subbands separated by guard bands (also referred to as the blind regions). Fig. 2 illustrates a particular case with $K=2$ subbands. Note that the bandwidth of each subband or guard band can differ.

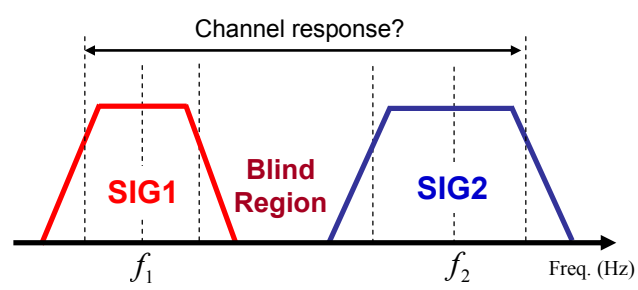

Fig. 2. Illustration of measurement channel for $K=2$.

As shown in Fig. 1, a sounding signal comprised of $G$ repeated pseudo-noise (PN) sequences is first up-sampled before being fed into a pulse shaping filter $h_{T}(t)$ such as a square-root raised cosine filter. After that, the pulsed-shaped signal is up-converted to $f_{k}$ and transmitted through the $k$-th subband. As a result, the transmit signal $s(t)$ is a superposition of multiple narrowband sounding signals residing in different subbands.

We consider a frequency-selective channel comprised of $L$ discrete MPCs (see Sec. V for a discussion of this assumption).
Thus, the channel impulse response can be expressed as

$$
h(t)=\sum_{\ell=1}^{L} \alpha_{\ell} \cdot \delta\left(t-\tau_{\ell}\right),
$$

where $\delta(\cdot)$ is the delta function while $\alpha_{\ell}$ and $\tau_{\ell}$ are the path gain and delay of the $\ell$-th MPC, respectively. Note that we have implicitly assumed that the channel remains approximately static over the $G$ PN sequences. The receiver obtains $r(t)$, the convolution of the transmit signal $s(t)$ with this complex channel impulse response $h(t)$, plus additive white Gaussian noise $w(t)$ that is modeled as a circularly symmetric complex Gaussian variable with standard deviation $\sigma$.

Upon receiving $r(t)$, the receiver first down-converts the received signal into each subband followed by matched filtering the down-converted signals with $h_{R}(t)$. The resulting $k$-th subband output is $y^{(k)}(t)$, for $k=1,2, \cdots, K$. Denote by $H(f)$ the frequency response of $h(t)$. Clearly, a straightforward least-squared (LS) estimate of $H(f)$ can be derived as follows.

$$
\hat{H}(f)=\frac{R(f)}{S(f)},
$$

where $R(f)$ and $S(f)$ are the Fourier transforms of $r(t)$ and $s(t)$, respectively. However, as shown in the later simulation, since $S(f) \approx 0$ over the blind region, the estimate $\hat{H}(f)$ derived from (2) will incur substantial estimation errors over the blind region. Note that this conventional method can be slightly improved by linear (or other) interpolation-based techniques between the measured subchannels. However, the improvement is minor if the width of the blind region is larger than the coherence bandwidth of the channel. In the sequel, the method shown in (2) is referred to as the conventional method.

In the next section, we will propose a super-resolution blind algorithm to derive the channel frequency response $H(f)$ by exploiting sounding signals in disjoint subbands. For presentational clarity, we will concentrate on the case of $K=2$ as illustrated in Fig. 2 in the sequel. However, it should be emphasized that the following discussion can be extended to $K>2$ in a straightforward manner. 


\section{Proposed Algorithm}

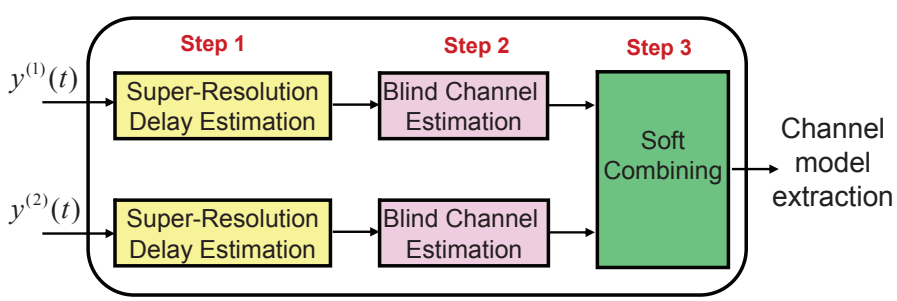

Fig. 3. Block diagram of the proposed super-resolution blind channel modeling algorithm

Fig. 3 illustrates the block diagram of the proposed superresolution blind channel modeling algorithm.

\section{A. Step One: Super-resolution delay estimation}

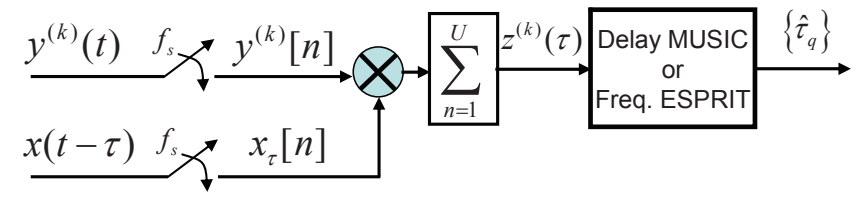

Fig. 4. Super-resolution delay estimation by exploiting $y^{(k)}(t)$.

In the first step, super-resolution delay estimation is performed by exploiting either delay-domain MUSIC [3] or frequency-domain ESPRIT [4]. Denote by $T_{c}$ and $U$ the PN sequence chip duration and the number of chips per PN sequence, respectively. In contrast to the conventional PN correlation method in which the resolution of path delay estimation is limited by $T_{c}$, the super-resolution delay estimation proposed in [3], [4] can provide estimates of resolution of a fraction of $T_{c}$. In particular, the ESPRIT algorithm is more computationally advantageous than MUSIC since it does not require exhaustive search [4].

Next, we propose an improved ESPRIT algorithm based on [4]. Two key differences distinguish the improved algorithm from [4]: (1) we need to take the pulse shaping into account; (2) rather than directly applying ESPRIT to the received signal as proposed in [4], we consider applying the ESPRIT algorithm after correlating the received signal with the transmitted PN sequence. Let $y^{(k)}(t)$ and $\tau$-delayed $x(t)$ denote first $D$ time oversampled at $f_{s}=1 /\left(D T_{c}\right)$ as shown in Fig. 4. Then, $y^{(k)}[n]$ is correlated with $x_{\tau}[n]$ and summed over one PN sequence. The resulting $z^{(k)}(\tau)$ takes the following form

$$
z^{(k)}(\tau)=\sum_{\ell=1}^{L} \alpha_{\ell} \cdot e^{-j 2 \pi \tau_{\ell} f_{k}} \cdot v(\tau)+\psi^{(k)}(\tau),
$$

where $v(\tau)$ is the autocorrelation function of the pulse-shaped PN sequence and $\psi^{(k)}(\tau)$ is the additive noise after correlation. Fig. 5 illustrates the function $v(\tau)$ for raised cosine pulseshaped PN sequences with different values of rolloff factor $\beta$. It is interesting to observe from Fig. 5 that the autocorrelation function associated with a smaller $\beta$ entails larger ripples outside $[-1,+1]$ as compared to the ideal autocorrelation function. In other words, a smaller rolloff factor results in better band-limiting performance at the cost of more interference for super-resolution delay estimation.

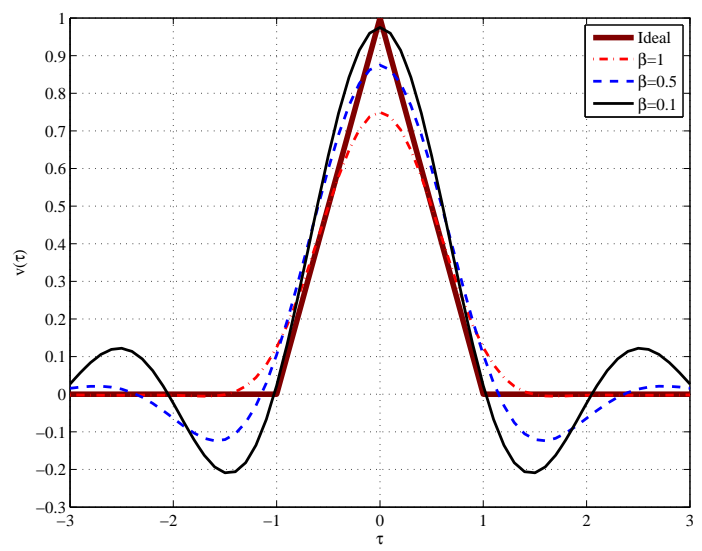

Fig. 5. Autocorrelation functions of raised cosine pulse-shaped PN sequence with different values of rolloff factor $\beta$.

Then, we convert $z^{(k)}(\tau)$ into the frequency domain before performing the frequency ESPRIT as follows. After deconvolution, we have

$$
J^{(k)}(f)=\frac{Z^{(k)}(f)}{V(f)}=\sum_{\ell=1}^{L} \alpha_{\ell} \cdot e^{-j 2 \pi \tau_{\ell} f_{k}}+\Xi^{(k)}(f),
$$

where $\Xi^{(k)}(f)=\frac{\Psi^{(k)}(f)}{V(f)}$ with $Z^{(k)}(f), V(f)$ and $\Psi^{(k)}(f)$ being the Fourier transforms of $z^{(k)}(\tau), v(\tau)$ and $\psi^{(k)}(\tau)$, respectively. $N$ samples of $J^{(k)}(f)$ are taken from its main lobe at $f=0, \Delta, 2 \Delta, \cdots,(N-1) \Delta$. It can be shown that the noise correlation matrix is given by

$$
\left[\boldsymbol{R}_{\Xi^{(k)}}\right]_{p, q}=\frac{\sigma^{2} \cdot \boldsymbol{F}_{N}(p,:) \cdot \boldsymbol{R}_{0} \cdot \boldsymbol{F}_{N}^{H}(q,:)}{|V(p \Delta)|^{2}},
$$

where $0 \leq p, q \leq N-1$ and $\boldsymbol{R}_{0}$ is the pulse-shaped noise covariance matrix with $\left[\boldsymbol{R}_{0}\right]_{p, q}=v\left(\tau_{p}-\tau_{q}\right)$. Substituting (5) into the frequency-domain ESPRIT algorithm in [4], we can extract super-resolution estimates of path delays denoted by by $\left\{\hat{\tau}_{q}^{(k)}\right\}$, where $q=1,2, \cdots, Q$ with $Q \geq L$.

\section{B. Step Two: Blind channel estimation}

Upon attaining $\left\{\hat{\tau}_{q}^{(k)}\right\}$, two approaches can be utilized to derive the MPC amplitudes and thus the channel impulse response, namely delay-domain and frequency-domain approaches. In the delay-domain approach, we first collect $I$ samples at the correlator output before forming a vector $\boldsymbol{z}^{(k)}=\left[\begin{array}{llll}z^{(k)}\left(T_{1}\right) & z^{(k)}\left(T_{2}\right) & \ldots & z^{(k)}\left(T_{I}\right)\end{array}\right]^{T}$. From (3), it is straightforward to show that $\boldsymbol{z}^{(k)}$ can be rewritten in the following matrix form:

$$
\begin{aligned}
& \boldsymbol{z}^{(k)}=\boldsymbol{B}(\boldsymbol{\tau}) \cdot \boldsymbol{\alpha}+\Psi^{(k)} \\
& \text { where } \begin{array}{lllll}
\boldsymbol{\alpha} & = & {\left[\begin{array}{llll}
\alpha_{1} & \alpha_{2} & \ldots & \alpha_{L}
\end{array}\right]^{T}, \quad \boldsymbol{B}(\boldsymbol{\tau})=} \\
{\left[\begin{array}{lllll}
\boldsymbol{v}\left(\tau_{1}\right) & \boldsymbol{v}\left(\tau_{2}\right) \ldots & \boldsymbol{v}\left(\tau_{L}\right)
\end{array}\right]} & \text { and } & \boldsymbol{v}\left(\tau_{\ell}\right) \\
{\left[\begin{array}{lllll}
v\left(T_{1}-\tau_{\ell}\right) & v\left(T_{2}-\tau_{\ell}\right) & \ldots & v\left(T_{I}-\tau_{\ell}\right)
\end{array}\right]^{T} .}
\end{array}
\end{aligned}
$$


As a result, the LS estimate of $\boldsymbol{\alpha}$ can be derived as

$$
\hat{\boldsymbol{\alpha}}=[\boldsymbol{B}(\hat{\boldsymbol{\tau}})]^{\dagger} \boldsymbol{z}^{(k)} .
$$

However, one possible drawback associated with the delaydomain approach is that the channel frequency response derived from (7) may exhibit large deviation from that estimated in (2) over the $k$-th subband.

Thus motivated, we next discuss the frequency-domain approach that extracts the channel amplitudes by exploiting the estimates derived from (2). This approach is shown to provide more accurate frequency response estimation over each measurement subband. We first define the channel impulse response vector as $\boldsymbol{h}_{N}=\left[h_{0}, h_{1}, \cdots, h_{N-1}\right]^{T}$ where only $L$ elements are non-zero. In the following, we exploit the fact that

$$
\boldsymbol{H}(f)=\boldsymbol{F}_{N} \cdot \boldsymbol{h}_{N}=\boldsymbol{F}_{N} \cdot \boldsymbol{T} \cdot \boldsymbol{h}_{L}^{\prime},
$$

where $\boldsymbol{h}_{L}^{\prime}$ contains only the $L$ non-zero elements of $\boldsymbol{h}_{N}$ and $\boldsymbol{T}$ is an $N \times L$ matrix whose $\ell$-th column is the $\left\lfloor f_{s} \tau_{\ell}\right\rfloor$-th column of $\boldsymbol{I}_{N}$. Thus, $\boldsymbol{F}_{N} \cdot \boldsymbol{T}$ is a sub-matrix of $\boldsymbol{F}_{N}$ with only the corresponding columns. Since $\left\{\tau_{\ell}\right\}$ is not available, we replace $\left\{\tau_{\ell}\right\}$ with $\left\{\hat{\tau}_{q}^{(k)}\right\}$ and (8) becomes

$$
\hat{\boldsymbol{H}}^{(k)}(f)=\boldsymbol{F}_{N} \cdot \boldsymbol{T}^{(k)} \cdot \boldsymbol{h}_{Q}^{\prime(k)}+\boldsymbol{\eta},
$$

where $\boldsymbol{\eta}$ is the additive noise and $\boldsymbol{T}^{(k)}$ is an $N \times Q$ matrix whose $q$-th column is the $\left\lfloor f_{s} \hat{\tau}_{q}^{(k)}\right\rfloor$-th column of $\boldsymbol{I}_{N}$. Thus, we have

$$
\hat{\boldsymbol{h}}_{Q}^{\prime(k)}=\left[\boldsymbol{F}_{N} \cdot \boldsymbol{T}^{(k)}\right]^{\dagger} \hat{\boldsymbol{H}}^{(k)}(f) .
$$

However, recall that estimates of $\hat{\boldsymbol{H}}^{(k)}(f)$ derived from (2) are reliable only over the $k$-th subband. Thus, in (10), we should take $M^{(k)}>Q$ samples of $\hat{\boldsymbol{H}}^{(k)}(f)$ only over the $k$ th subband derived from (2). Finally, substitution of $\hat{\boldsymbol{h}}_{Q}^{\prime(k)}$ into (9) results in $\hat{\boldsymbol{H}}^{(k)}(f)$ over the whole channel bandwidth.

\section{Step Three: Soft combining}

The last step is to combine $\hat{\boldsymbol{H}}^{(k)}, k=1,2$, to provide an accurate channel estimate over the whole wideband channel. Clearly, the resulting estimate has to satisfy at least the following two requirements. First of all, the combined estimate should render a continuous frequency response over the whole channel. Second, the combined estimate should provide good estimates over the blind regions as well as the measurement subbands. A soft-combining approach can be established as follows:

$$
\hat{\boldsymbol{H}}(f)=\sum_{k=1}^{K} \rho_{k}(f) \cdot \hat{\boldsymbol{H}}^{(k)}(f),
$$

where $\rho_{k}(f) \geq 0$ are the weighting coefficients at frequency $f$ with $\sum \rho_{k}^{2}(f)=1$. It is easy to see that $\left\{\rho_{k}(f)\right\}$ should be designed to accurately reflect the reliability of $\hat{\boldsymbol{H}}^{(k)}(f)$. Note that $\hat{\boldsymbol{H}}^{(k)}(f)$ becomes less reliable as $f$ falls far from the $k$-th subband. Inspired by this observation, a simple but effective design example of $\left\{\rho_{k}(f)\right\}$ is shown in Fig. 6 where $\rho_{k}(f)$ remains unity over the $k$-th subband and linearly decreases to zero over the blind region. Alternative combination methods that aim to minimize the mean-square error of the estimate at each frequency will be described in our future works.

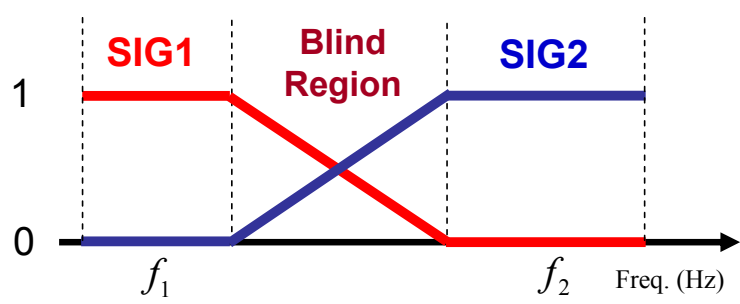

Fig. 6. Examples of weighting coefficients employed for soft combining.

\section{Simulation Results}

In this section, simulation results are shown to demonstrate the performance of the proposed super-resolution blind algorithm. As shown in Fig. 1, we simulate a system of $K=2$ equal-bandwidth subbands with $f_{1}=0 \mathrm{~Hz}$ and $f_{2}=2$ $\mathrm{Hz}$, i.e. both subbands and their guard band have bandwidth of $1 \mathrm{~Hz}$. The received signal is 8-time oversampled. Thus, the subband $\# 1$, subband $\# 2$ and the blind region reside in the following normalized frequency band (with respect to the sampling frequency), $\left[-\frac{1}{16},+\frac{1}{16}\right],\left[+\frac{1}{16},+\frac{3}{16}\right]$ and $\left[+\frac{3}{16},+\frac{5}{16}\right]$, respectively. Unless otherwise specified, raised cosine pulse shaping with $\beta=0.5$ is employed in the following simulation. Furthermore, for illustration purposes, a frequency-selective complex fading channel with $L=5$ paths is simulated. The path delays normalized with respect to $T_{c}$ are set to $\tau=\{1.0,1.6,2.4,3.1,4.0\}$ whereas the path gains are randomly generated in each simulation run with path power of $\{0,0,0,-3,-3\} \mathrm{dB}$. It is worth noting that the conventional correlation-based delay estimation approach will fail under this simulation setup since there are MPCs separated by less than one $T_{c}$.

Fig. 7 depicts the estimated channel frequency response obtained with the conventional method as shown in (2). Inspection of Fig. 7 suggests that the conventional method incurs substantial estimation errors over the blind region as well as the edges of the signal subbands due to raised cosine pulse shaping. Indeed, the peak shown in the blind region is always present, regardless of the underlying channel model. This is because that the peak is induced by $S(f) \approx 0$ in (2) over the blind region.

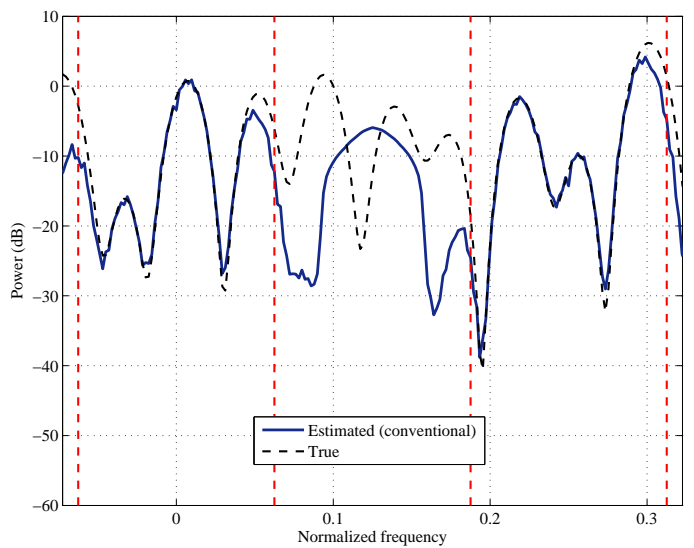

Fig. 7. Performance of the conventional channel estimation method. 
Fig. 8 shows the estimated $\hat{\boldsymbol{H}}^{(2)}$ using the sounding signal in subband \#2. While Fig. 8 shows improved estimates of $\hat{\boldsymbol{H}}^{(2)}$ over the blind region as compared to Fig. 7, the estimation performance degrades rapidly beyond subband \#2. Similar observation can be also obtained from the estimated $\hat{\boldsymbol{H}}^{(1)}$ derived from subband \#1.

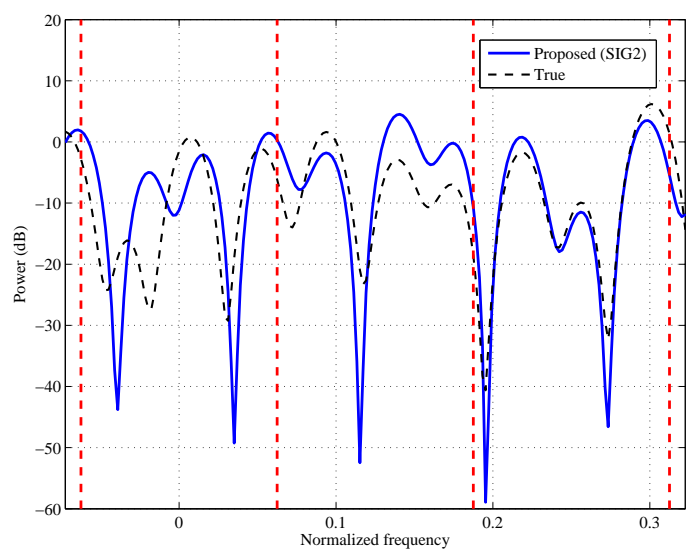

Fig. 8. Performance of the proposed super-resolution blind algorithm by exploiting received signal from subband \#2.

Next, Fig. 9 shows the estimated $\hat{\boldsymbol{H}}$ by combining $\hat{\boldsymbol{H}}^{(1)}$ and $\hat{\boldsymbol{H}}^{(2)}$ using the weighting coefficients shown in Fig. 6. Compared to Fig. 8, Fig. 9 indicates that the proposed superresolution blind algorithm can provide balanced good performance over both measurement subbands and the blind region.

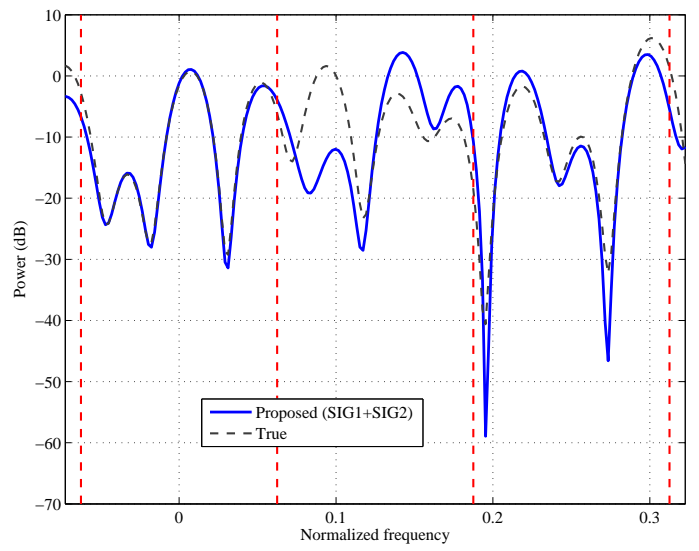

Fig. 9. Performance of the proposed super-resolution blind algorithm after combining both two subbands.

Finally, Fig. 10 shows the performance of the conventional method and the proposed super-resolution blind channel modeling algorithm in terms of relative mean squared error (RMSE) over the blind region as a function of signal-tonoise ratio (SNR) defined as $1 / \sigma^{2}$. More specifically, we define $R M S E=E\left\{\frac{|\hat{\boldsymbol{H}}-\boldsymbol{H}|^{2}}{|\boldsymbol{H}|^{2}}\right\}$. The results are averages over 1000 runs. Fig. 10 shows that the proposed algorithm substantially outperforms the conventional method. This can be explained by the fact that the conventional method always estimates a peak over the blind region, regardless of the actual channel. However, it is very likely that null channel responses exist over the blind region. As a result, the RMSE of the conventional method is dominated by large errors due to error magnifications over the null responses by dividing zero. Furthermore, we can observe that the performance of the proposed algorithm is insensitive to SNRs.

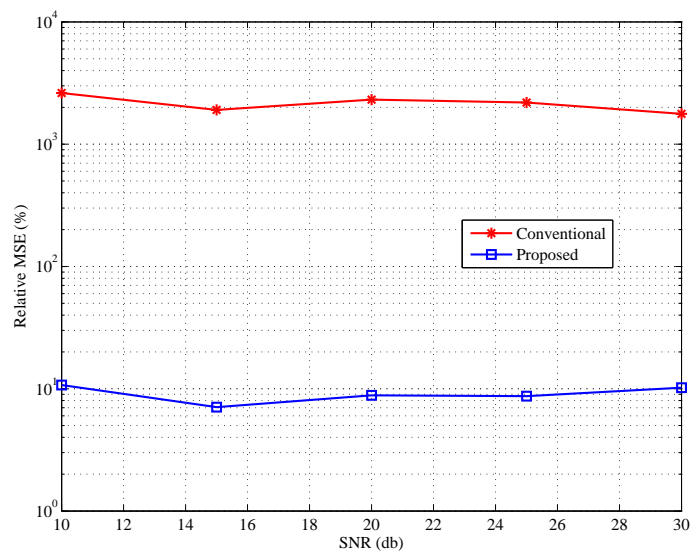

Fig. 10. RMSE as a function of $S N R$ over time-invariant multipath fading channels.

\section{CONCLUDING REMARKS}

In this paper, we have presented a method for reconstructing propagation channels from measurements in disjoint subbands of the band of interest. By using high-resolution estimation of the multipath parameters, and suitable combining of the results, we arrived at a model that accurately interpolates between the measured subbands. The methodology was verified by means of a synthetic channel model, where the "correct" description was exactly known.

There are several extensions of this study that can be further explored. One fundamental assumption in this work is the validity of the tapped-delay-line representation shown in (1) with a finite number of taps. Therefore, for channels of very large bandwidth and/or diffuse MPCs, it is important to develop effective means to confirm the validity of this assumption before applying the proposed algorithm [5]. Furthermore, the performance of the proposed algorithm hinges on the accuracy of super-resolution delay estimation. Thus, it deserves further investigation on improving the delay estimation accuracy. Finally, tracking the estimated channel model over time may help identify and remove channel modeling artifacts due to estimation errors.

\section{REFERENCES}

[1] M. Ibnkahla (ed.), Digital Signal Processing for Wireless Communications Handbook. CRC Press, 2004.

[2] A. Molisch, M. Shafi, and L. J. Greenstein, "Propagation issues for cognitive radio," Proceedings of the IEEE, vol. 97, pp. 787-804, March 2009.

[3] T. Manabe and H. Takai, "Superresolution of multipath delay profiles measured by PN correlation method," IEEE Trans. on Antennas and Propagation, vol. 40, pp. 500-509, May 1992.

[4] H. Saarnisaari, "TLS-ESPRIT in a time delay estimation," in Proc. IEEE 47th Vehicular Technology Conference, Phoenix, AZ, May 1997.

[5] P. A. Bello, "Characterisation of randomly time-variant linear channels," IEEE Trans. Commun. Systems, vol. CS-11, pp. 360-393, Dec. 1963. 